\title{
Use of an antiviral filter attached to a pleural drain bottle to prevent aerosol contamination with SARS-CoV-2
}

\author{
Authors: Mohammed R Akhtar, ${ }^{A}$ William Ricketts ${ }^{B}$ and Tim Fotheringham ${ }^{A}$
}

Pneumothoraces (1\%) and pleural effusions (5\%) are two of the less common complications of infection with COVID-19. Following a referral for a pleural drain insertion for a pneumothorax in a patient with COVID-19, we reassessed the infection risks involved in this procedure and its aftercare. Pleural drainage tubes attached to an underwater seal drain allow expulsion of aerosol and larger droplets via the vent from the bottle into the surrounding environment, potentially leading to infection of other patients and staff.

Consequently, we chose to attach an antiviral filter to the venting port of an underwater seal drain bottle to mitigate this risk. A fluorescein dye experiment was used to demonstrate the reduction in aerosol emission output from the bottle with our described technique, allowing an antiviral filter to be attached to a pleural underwater seal drainage bottle for added protection of patients and staff in the local environment.

KEYWORDS: Coronavirus, COVID-19, pneumothorax, chest drain, pleural

DOI: $10.7861 /$ clinmed.2020-0246

Pneumothoraces (1\%) and pleural effusions (5\%) are two of the less common complications of infection with COVID-19.1,2 Following a referral for a pleural drain insertion for a pneumothorax in a patient with COVID-19 (Fig 1), we reassessed the infection risks involved in this procedure and its aftercare. Pleural drainage tubes attached to an underwater seal drain allow expulsion of aerosol and larger droplets via the vent from the bottle into the surrounding environment, potentially leading to infection of other patients and staff.

Consequently, we chose to attach an antiviral filter to the venting port of an underwater seal drain bottle to mitigate this risk. The antiviral filter attached is a disposable filter from an anaesthetic machine rated at $>99.99 \%$ filtration efficiency (Clear-Guard ${ }^{\mathrm{TM}} 3$ breathing filter, Intersurgical Ltd). The filter was attached to $6 \mathrm{~mm}$ oxygen tubing using a straight $22 \mathrm{~mm}$ to $6 \mathrm{~mm}$ connector. Approximately $2.5 \mathrm{~cm}$ of oxygen tubing was used to attach the filter to the vent port on the drain bottle and secured with cable ties (Figs 2 and 3).

Authors: ${ }^{A}$ consultant interventional radiologist, Barts Health NHS Trust, London, UK; ${ }^{\mathrm{B}}$ Consultant chest physician, Barts Health NHS Trust, London, UK

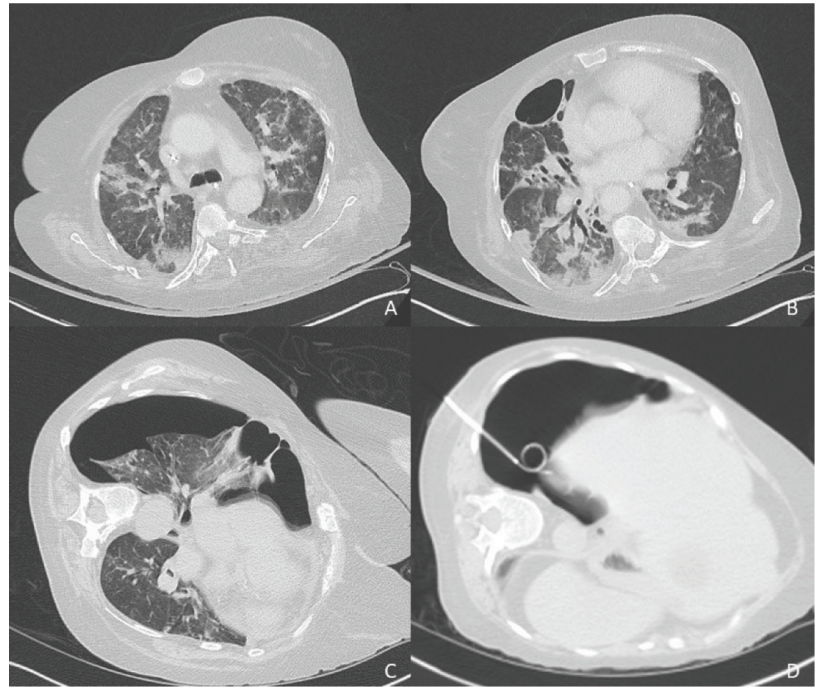

Fig 1. Computed tomography prior to pneumothorax showing infiltrates in keeping with COVID-19 infection $(a, b)$ and right upper lobe bulla (b), with pneumothorax immediately prior to (c) and immediately after (d) insertion of pleural drain.

To demonstrate the reduction in aerosol emission output from the bottle, $1 \mathrm{~g}$ of fluorescein dye was added to $500 \mathrm{~mL}$ of water in a standard underwater seal drain bottle. The pleural drain was connected to room air at a flow rate of $5 \mathrm{~L} / \mathrm{min}$. A black card was

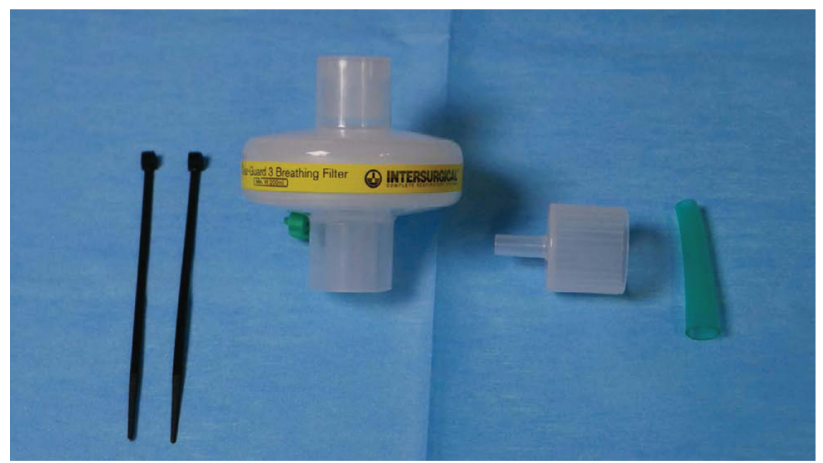

Fig 2. Components used to connect antiviral filter to the underwater seal vent. From left to right: cable ties, antiviral filter (Clear-Guard ${ }^{\mathrm{TM}} 3$ breathing filter, 1544000 , Intersurgical Ltd), straight connector $22 \mathrm{~F}$ to $6 \mathrm{~mm}$ oxygen stem (15680000, Intersurgical Ltd) and oxygen tubing. 


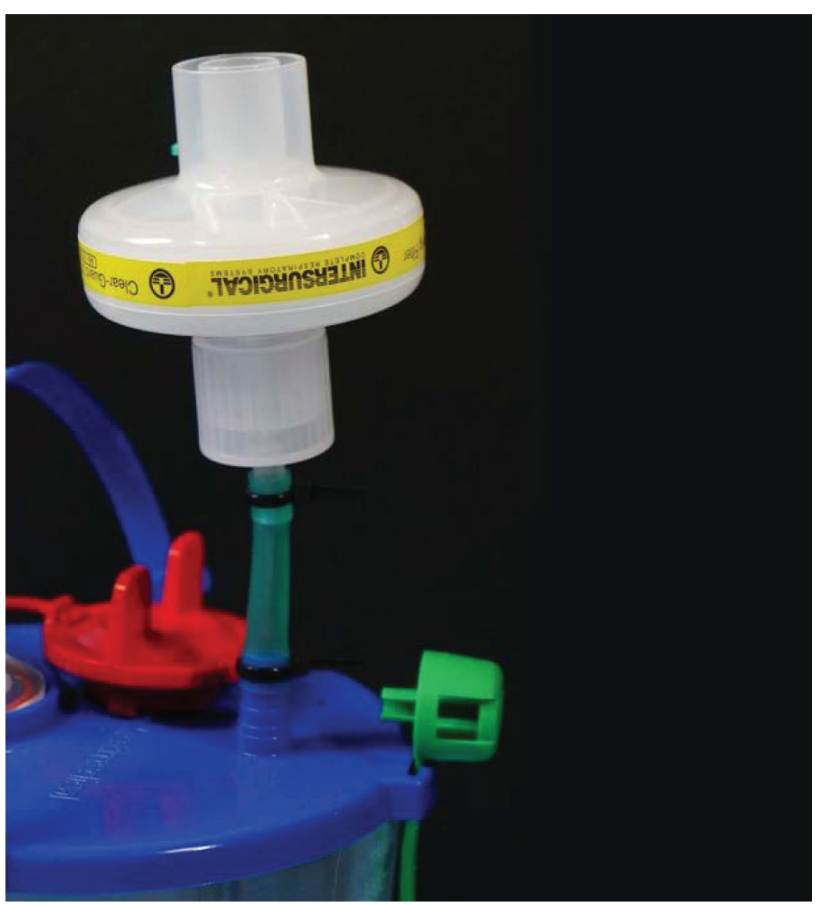

Fig 3. Underwater seal drain bottle (R54500 Rocket blue single bottle system, Rocket Medical) connected to the antiviral filter.

placed $3 \mathrm{~cm}$ above the bottle vent and photographed under a UV light after 2 hours. The experiment was repeated without and with the filter attached, and photographs taken demonstrate aerosol emissions and droplets from the drain bottle when the filter is not used (Fig 4).

Possible aerosol contamination from a pleural underwater seal drain merits further investigation. A significant proportion of COVID-19 patients are on higher oxygen requirements or positive pressure ventilation with the pleural drain in situ for longer than the 2 hours we allowed for in our simulation, leading to potentially even greater aerosol loads being generated. This simulation was not validated for speed or size of particles expelled through the venting port. Despite this, our described technique, which utilises disposable equipment that is readily available, allows an antiviral filter to be attached to a pleural underwater seal drainage bottle for added protection of patients and staff in the local environment.

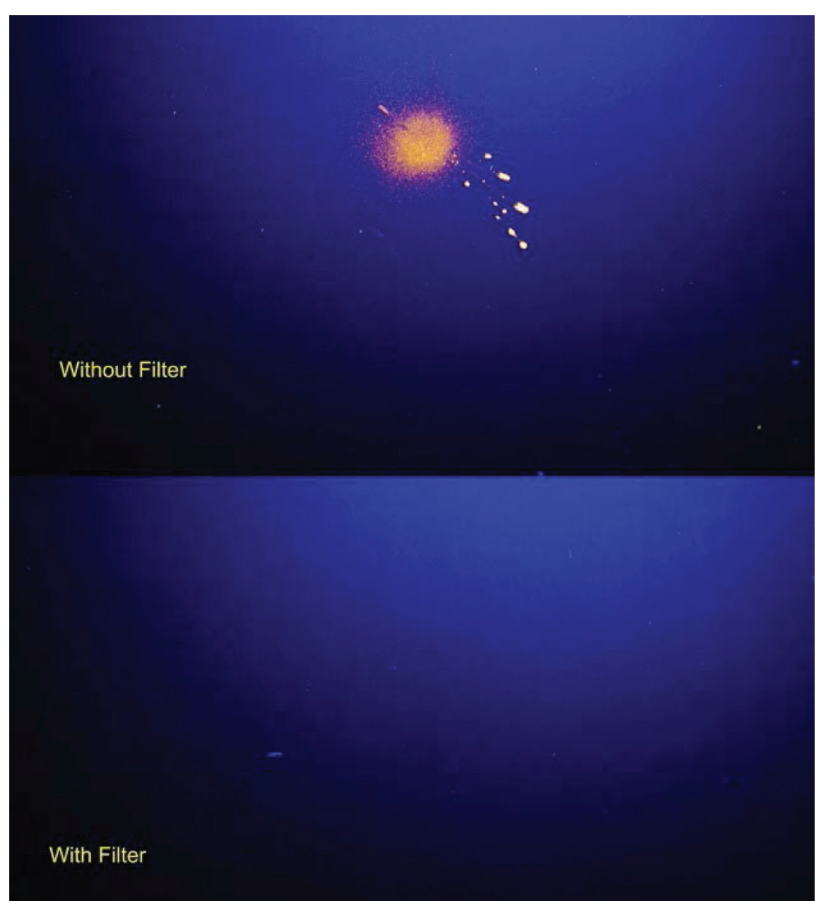

Fig 4. Fluorescein dye expelled from an underwater seal drain vent onto black card $3 \mathrm{~cm}$ above the vent.

\section{References}

1 Shi $\mathrm{H}$, Han X, Nanchuan ] et al. Radiological findings from 81 patients with COVID-19 pneumonia in Wuhan, China: a descriptive study. Lancet Infect Dis 2020;20:425-34.

2 Chen N, Zhou M, Dong X et al. Epidemiological and clinical characteristics of 99 cases of 2019 novel coronavirus pneumonia in Wuhan, China: a descriptive study. Lancet 2020;395:507-13.

Address for correspondence: Dr William Ricketts, Department of Respiratory Medicine, St Bartholomew's Hospital, West Smithfield, London EC1A 7BE, UK.

Email: william.ricketts@nhs.net 